\title{
Magnetic phase transition in confined MnO nanoparticles studied by polarized neutron scattering
}

\author{
Mikhail Feygenson, ${ }^{1}$ Werner Schweika, ${ }^{2}$ Alexander Ioffe, ${ }^{2}$ Sergey B. Vakhrushev, ${ }^{3}$ and Thomas Brückel ${ }^{2}$ \\ ${ }^{1}$ Condensed Matter Physics and Materials Science Department, Brookhaven National Laboratory, Upton, New York 11973, USA \\ ${ }^{2}$ Institut für Festkörperforschung, Forschungszentrum Jülich GmbH, 52425 Jülich, Germany \\ ${ }^{3}$ Ioffe Physico-Technical Institute, St. Petersburg 194021, Russia
}

(Received 30 November 2009; revised manuscript received 2 February 2010; published 22 February 2010)

\begin{abstract}
We have investigated the magnetic ordering and the phase transition in $\mathrm{MnO}$ nanoparticles confined in a porous glass using polarized neutron scattering. These $\mathrm{MnO}$ nanoparticles are best described as extended wormlike structures with a mean diameter of $70 \AA$. We observe an apparent continuous magnetic phase transition in $\mathrm{MnO}$ nanoparticles, in contrast to the well-known discontinuous phase transition in bulk MnO. By polarization analysis, separating the magnetic scattering, it is found that within the individual MnO nanoparticles about $60 \%$ of atoms remain disordered in the low-temperature limit, presumably due to interactions between nanoparticles and glass walls. The continuous character of the phase transition and the unusual temperature dependence suggests a surface-induced disorder phenomenon.
\end{abstract}

DOI: $10.1103 /$ PhysRevB.81.064423

PACS number(s): 64.70.Nd, 28.20.Cz, 52.55.-s

\section{INTRODUCTION}

Magnetic nanoparticles are currently in the focus of intensive fundamental research for future information technology and an improved understanding of modified or even new phenomena not previously observed in their bulk counterparts are of crucial interest. While finite-size effects on magnetic properties of materials are relatively well documented, ${ }^{1,2}$ much less is known about effect of a nanoscale confinement on magnetic proprieties of materials. ${ }^{3-6}$ In confined geometries, finite-size effects are combined with material-dependent interactions with interfaces, which presumably can alter the thermodynamic and magnetic behavior. Our particular interest is the effect of nanoscale confinement on the magnetic phase transition in the classical antiferromagnet $\mathrm{MnO}$. In a previous study of confined $\mathrm{MnO}$ nanoparticles by unpolarized neutron scattering, ${ }^{3}$ two important observations have been made. First, the magnetic phase transition in confined $\mathrm{MnO}$ nanoparticles becomes apparently continuous in contrast to the first-order transition otherwise observed in bulk MnO. Second, the magnetic correlation length was substantially smaller than the average size of the $\mathrm{MnO}$ nanoparticles. Golosovsky et al. ${ }^{3}$ have suggested the following explanations. The rounding of the magnetic phase transition was ascribed to the finite-size effects while the reduction in magnetic correlation length was a consequence of the random canting of spins at the $\mathrm{MnO}$ nanoparticle/glass interface.

In order to stimulate a further discussion on magnetic phase transitions on nanoscale, we carried out polarized neutron diffraction experiments on confined $\mathrm{MnO}$ nanoparticles. Polarization analysis enables the direct observation of the previously suggested spin-canting effect in $\mathrm{MnO}$ nanoparticles, as well as it allows to separate a relatively weak magnetic scattering from a large nonmagnetic scattering background from the glass matrix. In agreement with previous results of Golosovsky et al., we have observed the apparent absence of a discontinuous transition in $\mathrm{MnO}$ nanoparticles confined in nanopores. Here, we include in our consider- ations effects of surface-induced disorder, in which the $\mathrm{MnO} / \mathrm{glass}$ interface is wetted by the disordered paramagnetic phase. ${ }^{7-12}$ The proposed model obviously addresses the effect of the nanoconfinement on the magnetic properties of $\mathrm{MnO}$, in contrast to the previous explanation where only finite-size effects were considered. ${ }^{3}$ While finite-size effects certainly exist and contribute to a rounding of the phase transition, mere finite-size effects are insufficient to explain the lowering of the order parameter observed over the whole temperature range and indicate a balance between a near surface disordered film and the inner part of the ordered $\mathrm{MnO}$ nanoparticle.

\section{SAMPLE AND EXPERIMENT RESULTS}

The porous matrix was made from a sodium borosilicate glass and it consists of pure $\mathrm{SiO}_{2}$ with a random interconnected network of elongated pores of $70 \AA$ diameter. The pore size is extremely uniform with a size distribution width of less then 3\%. The $\mathrm{MnO}$ was synthesized within the nanopores from a manganese nitrate solution by a chemical bath deposition method, without applying external pressure inside the glass matrix. More details on the sample preparation can be found elsewhere. ${ }^{3,13}$

To study the magnetic phase transition in our sample, we used the diffuse neutron scattering (DNS) instrument at DIDO reactor FRJ-2 in Forschungszentrum Jülich, Germany, ${ }^{14}$ set at a neutron wavelength of $5.2 \AA$. The nanopore sample and the bulk powder $\mathrm{MnO}$ sample was measured in a standard thin-walled $\mathrm{Al}$ container as a sample holder.

In order to separate the magnetic neutron scattering from $\mathrm{MnO}$ nanoparticles from the scattering of the glass matrix and also from the spin-incoherent scattering background on Mn, we have performed experiments with polarization analysis along three orthogonal directions (Fig. 1). The separated magnetic neutron scattering clearly displays the magnetic Bragg peak $\left(\frac{1}{2} \frac{1}{2} \frac{1}{2}\right)$ at low temperatures and further reveals that a part of the atoms in a $\mathrm{MnO}$ nanoparticle remains dis- 


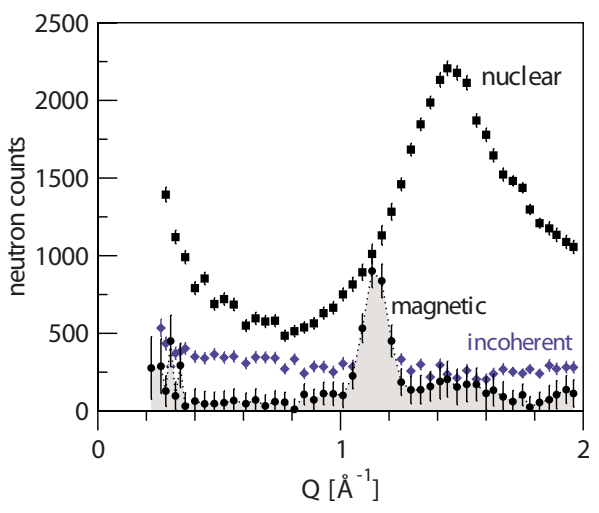

FIG. 1. (Color online) The separation of nuclear coherent, nuclear incoherent, and purely magnetic scattering by polarization analysis for confined $\mathrm{MnO}$ nanoparticles at $10 \mathrm{~K}$.

ordered even at very low temperatures, resulting in a relatively flat diffuse background. One easily notices that the magnetic scattering is weak compared to the nuclear scattering contribution from the glass matrix. Such a diffuse background due to magnetic disorder has been proposed earlier by Golosovsky, however, could not be evidenced from their unpolarized scattering experiments. ${ }^{3}$ From the peak-tobackground ratio at $10 \mathrm{~K}$, we estimated that a fraction of $60 \% \mathrm{Mn}$ atoms in the $\mathrm{MnO}$ nanoparticle remains disordered, probably due to interactions with the glass walls, resulting in randomly canted spins near the surface. Furthermore, the temperature dependence of the magnetic $\left(\frac{1}{2} \frac{1}{2} \frac{1}{2}\right)$ Bragg peak has been measured in detail by spin-flip scattering in the range from 2 to $140 \mathrm{~K}$. The integrated intensities of these peaks were used to define the order parameters for both bulk and nanoparticles. Representative profiles of the magnetic $\left(\frac{1}{2} \frac{1}{2} \frac{1}{2}\right)$ Bragg peak for $\mathrm{MnO}$ nanoparticles at high and low temperatures are depicted in Fig. 2. The peak width is not due to resolution but results from the size of the ordered magnetic domains in the nanopores, which are smaller than the nanopore size, in agreement with results of Golosovsky et al. As a reference for the bulk properties, we also measured a $\mathrm{MnO}$ powder sample under the same experimental conditions.

The order parameters as a function of temperature for $\mathrm{MnO}$ nanoparticles and bulk powder are shown in Fig. 3.

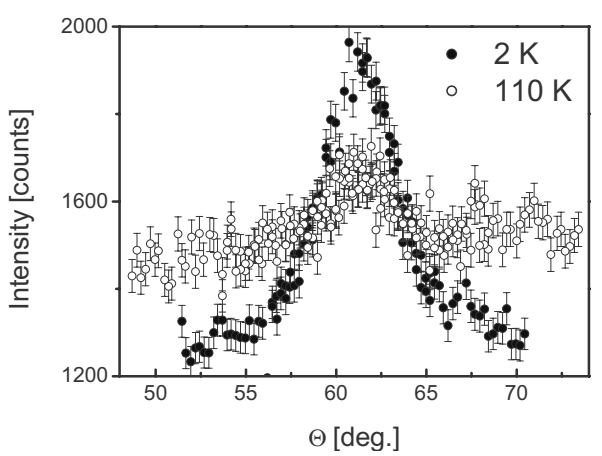

FIG. 2. The magnetic $\left(\frac{1}{2} \frac{1}{2} \frac{1}{2}\right)$ Bragg peak of the confined MnO nanoparticles at 2 and $110 \mathrm{~K}$. The peak is observed in the spin-flip channel and is not limited by resolution; the raw data contains both the incoherent and magnetic scattering.

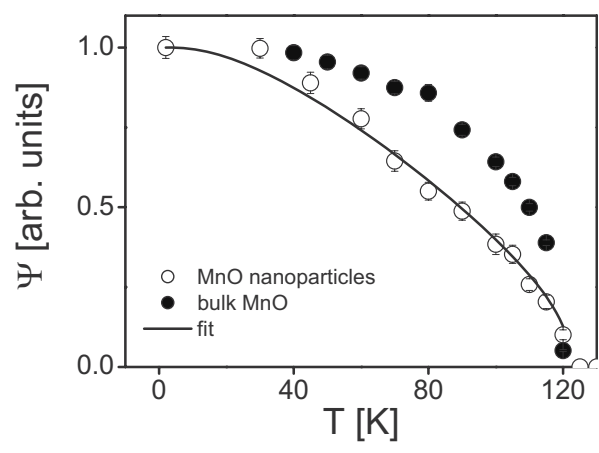

FIG. 3. The order parameter $\Psi$ for the $\mathrm{MnO}$ nanoparticles and bulk powder of $\mathrm{MnO}$. The solid line is a surface-induced disorder model for $\mathrm{MnO}$ nanoparticles (see text for details).

Apparently, the magnetic phase transition becomes continuous in $\mathrm{MnO}$ nanoparticles. The origin of this rounding will be discussed in details in the following sections. A closer look to the data indicates that the phase transition in $\mathrm{MnO}$ nanoparticle occurs at the Néel temperature of $T_{N}=122 \mathrm{~K}$, which is slightly higher then the Néel temperature of $119 \mathrm{~K}$ of the bulk MnO. The similar effect was observed by Golosovsky and co-workers.

\section{DATA ANALYSIS}

Considering continuous magnetic phase transitions on nanoscale, a first approach is to apply finite-size scaling corrections to conventional critical exponents. ${ }^{15-17}$ However, here in case of a first-order phase transition in the bulk, this formalism is not adequate. Hence, we propose an alternative approach to describe the magnetic phase transition in confined $\mathrm{MnO}$ nanoparticles, by the theory of surface-induced disorder as derived for Ising spins and planar geometries. ${ }^{7-11}$ The model implies a situation when a layer of disordered phase gradually introduces at the surface of a nanoparticle. The layer further penetrates into the ordered bulk, consequently leading to the surface-induced disorder of the entire nanoparticle (Fig. 4). This mode ${ }^{11}$ was successfully used to describe the surface-induced magnetic phase transitions in $\mathrm{AB}$ binary alloys on a face-centered-cubic lattice.

We applied the same model to describe the experimental data for the following reasons. The results of the full polarization analysis clearly indicated a magnetic disorder even at

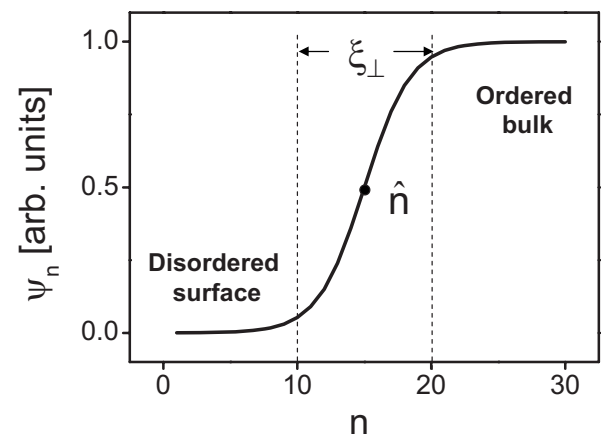

FIG. 4. A sketch of parameters used in the fit. 
low temperatures in $\mathrm{MnO}$ nanoparticles. We suggest that the disorder originates on the particle surface since the surface disorder in magnetic nanoparticles is well-documented phenomenon. ${ }^{18,19}$ It is plausible to assume that the interactions of $\mathrm{MnO}$ nanoparticles with the glass walls also contribute to the surface disorder. Thus, the surface-induced disordering of $\mathrm{MnO}$ nanoparticles seems as a very possible scenario. In case of planar geometry and surface-induced disorder, the disordered near surface film exhibits a weak and logarithmic singularity. Hence, a similar behavior of the order parameter can be expected, at least at intermediate temperatures, when the disordered film and the interfacial thickness, related to the correlation length in the bulk, is small compared to the nanopore diameter. In conclusive remarks, we will discuss limitations and imperfections of our model. Although, we cannot show and do not even expect that the model has the correct asymptotic behavior near the phase transition, we transfer this theoretical description in order to keep the number of independent parameters minimal. Actually, a single independent model parameter suffices to describe the temperature dependence of the order parameter of $\mathrm{MnO}$ nanoparticles.

The order parameter is comprised by the sum of the local order parameters in each atomic layer of an individual $\mathrm{MnO}$ nanoparticle,

$$
\Psi=\sum_{n} N_{n} \psi_{n}
$$

where $N_{n}$ is the number of atoms in atomic layer $n$ and $\psi_{n}$ is the order parameter in the same layer. We neglected the size distribution of $\mathrm{MnO}$ nanoparticles, due to the less then $3 \%$ polydispersity of the glass pores.

The order parameter in $n$th layer is expressed as follows:

$$
\psi_{n}=\psi_{\max }\left[1+\exp \left\{-2 \xi_{\perp}^{-1}(n-\hat{n})\right\}\right]^{-1},
$$

where $\hat{n}$ is the interface position, $\psi_{\max }=1$ is the maximum order parameter, and $\xi_{\perp}$ is the interfacial roughness. This tanh profile of the interface ${ }^{20}$ is expected to be a close approximation. The parameters are schematically depicted in the Fig. 4. For the three-dimensional systems, the critical behavior at the wetting transition depends on the value of dimensionless parameter $\omega$,

$$
\omega=k_{B} T_{c, b} /\left(4 \pi \widetilde{\Sigma} \xi_{d}^{2}\right),
$$

where $k_{B}$ is the Boltzmann constant, $T_{c, b}$ is the phasetransition temperature of the bulk, $\widetilde{\Sigma}$ is the interfacial stiffness between ordered and disordered phases, and $\xi_{d}$ is the correlation length of the order-parameter fluctuations in the disordered bulk phase.

Two limiting cases of interfacial stiffness have to be considered in our model. ${ }^{10}$ The description of the interface between ordered and disordered phases would be different for the large interfacial stiffness $(0<\omega \leq 1 / 2)$ and for the small interfacial stiffness $(\omega>1 / 2)$. The preliminary simulations indicated that the data is best described when $\omega>1 / 2$, i.e., the interface is rather smooth. Hence, the parameters in Eq. (2) can be calculated as
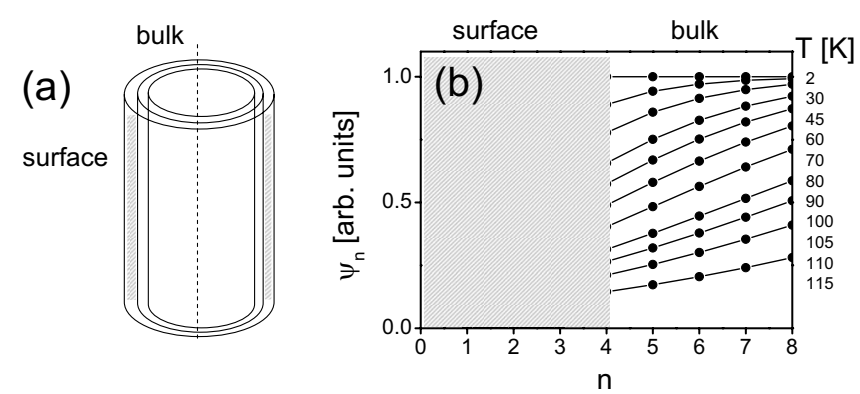

FIG. 5. (a) The sketch of the cylinder geometry and atomic layering. (b) The order-parameter $\psi_{n}$ profile versus atomic layer at different temperatures. The shaded area indicates three atomic layers which were excluded from the model. Solid lines are guide for the eyes.

$$
\begin{gathered}
\xi_{\perp}=\xi_{d} \sqrt{\omega}[\ln (1 / t)]^{1 / 2}, \\
\hat{n}=\sqrt{2 \omega} \ln (1 / t),
\end{gathered}
$$

where, $t=1-T / T_{c, b}$ is the reduced temperature.

The next step was to make an adequate estimation of the parameters in our model. We estimated the number of atomic layers $n$ assuming a cylindrical shape of the $\mathrm{MnO}$ nanoparticles with a radius of $35 \AA$ [Fig. 5(a)]. As a result, in our approximation, the cylindrical $\mathrm{MnO}$ nanoparticle had eight atomic layers, where the number of atoms $N_{n}$ in $n$th atomic layer was calculated using the bulk value of the $\mathrm{MnO}$ lattice constant $4.44 \AA .{ }^{21}$ The bulk correlation length $\xi_{d}=8.88 \AA$, determining the interfacial width, was estimated from the previous neutron diffraction study of the bulk MnO. ${ }^{21}$ The bulk transition temperature $T_{c, b}=119 \mathrm{~K}$ was obtained directly from our data (Fig. 3). The full polarization analysis indicated that about $60 \%$ of atoms in the $\mathrm{MnO}$ nanoparticle are disordered and thus cannot be described in the frame of the present model. It corresponds to approximately three atomic layers, thus we set $N_{1-3}=0$. It is a rather surprising result that three atomic layers are magnetically disordered. One possible explanation is the inhomogeneous interface between $\mathrm{MnO}$ nanoparticles and glass walls. Presumably, the interfacial roughnesses can lead to a disorder of more than one atomic layer on the surface of the $\mathrm{MnO}$ nanoparticle.

The number of atomic layers $n$, the number of atoms in each layer $N_{n}$, and the bulk correlation length $\xi_{d}$ have been fixed in the refinement procedure of the phase transition in $\mathrm{MnO}$ nanoparticles, which is finally modeled by a single free parameter $\omega$.

\section{RESULTS AND DISCUSSION}

The temperature dependence of the order parameter of confined $\mathrm{MnO}$ as compared to the bulk material reveals interesting features, a suppression of order over nearly the entire temperature range, an apparent continuous transition, and may be surprising at first sight, an enhanced temperature stability of the ordered phase. In the previous study by Golosovsky et al., a similar observation was made and the increase in the Néel temperature has been tentatively associated with the wetting of the glass walls by $\mathrm{MnO}$ 
nanoparticles. ${ }^{3}$ This picture, however, is difficult to accept because a pinning of random spin orientation by the nanopore wall should consequently results only in a lowering of order compared to situation in the infinite bulk.

There are two possible effects that are a more likely origin of the higher phase transition in $\mathrm{MnO}$ nanoparticles than in $\mathrm{MnO}$ bulk material. The first reason of this interesting observation could be a nanoconfinement-induced pressure in $\mathrm{MnO}$ nanoparticles. If one compares to the bulk powder of $\mathrm{MnO}$, studied by Yoo et al., ${ }^{22}$ the Néel temperature is gradually increasing with applied pressure. To discuss a probable second case, we recall the type of $\mathrm{MnO}$ order in the bulk, i.e., an alternating layering of spins in (111) planes, ${ }^{23}$ in which the competition between the four different ordering variants along the symmetrically equivalent directions leads to a firstorder phase transition similar to the four-state Potts model in case of Ising spins. One may speculate that the nanocylindrical geometry could prefer one ordering variant, in particular, an alternate layering along the cylinder axis, which would change the universality class and restore a continuous phase transition. Of course, the present powder-diffraction data do not provide such a deeper insight and we have to leave these aspects to future experiments and modeling.

The result of the model refinement for the temperature dependence of the order parameter is shown as a solid line in Fig. 3. The derived value of $\omega=6.8 \pm 0.57$ indicates a relatively smooth interface between order and disordered phases. We used this value of $\omega$ to calculate the local order parameter in each atomic layer of an individual $\mathrm{MnO}$ nanoparticle using Eqs. (4) and (2), respectively. As a result, the orderparameter profile of the $\mathrm{MnO}$ nanoparticle was reconstructed at different temperatures. Figure 5(b) indicates that the surface-induced disorder gradually penetrates into the inner part of $\mathrm{MnO}$ nanoparticles as temperature increases. At lowest temperature of $10 \mathrm{~K}$, the interior of $\mathrm{MnO}$ nanoparticle is ordered. When the temperature increases to $80 \mathrm{~K}$, the order parameter in each individual layer is reduced by at least factor of 2 . In vicinity of $T_{N}$, the order parameters reached their minima.

Our approach gives an atomic insight into order-disorder phase transition in nanoparticles, and it is entirely based on the fitting of only one free parameter. While all other variables were derived independently and they were fixed during the fit. An excellent agreement with experimental data supports our assumption that the disordered spins are located on the interface between $\mathrm{MnO}$ nanoparticles and glass walls.

Here, we used the theory of the surface-induced disorder for semi-infinite systems, by which however effects of finite curvature and shape on the phase transition were not taken into account. We assumed that the radius of curvature is infinitely large and basically described the wetting in the planar geometry. We next argue that it is a first step toward developing of the model, which describes the magnetic phase transition in confined geometries for cylindrical nanoparticles. To our knowledge, no model explicitly describing our case is available up to date.

The phase transitions have been intensively studied within past decades, however, the exact mechanism behind the order-disorder transition on nanoscale is still under debate. In part, it is explained by a very limited number of experimental results available today. Recent experiments on molecular aggregates of water revealed a size-dependent thermodynamics of free water. ${ }^{24}$ The situation becomes much different when the clusters of water are confined. Oguni et al. ${ }^{25}$ experimentally studied the glass transitions of water confined in silicagel nanopores of various sizes. They argued that the water confined in nanopores can be divided into two parts: interfacial water in contact to glass walls and internal water inside the pores. These two parts have very different dynamical properties. By changing the size of nanopores, it was possible to manipulate the ratio between interfacial and internal water. As a result, a glass transition was observed at temperatures depending on the size of the nanopores. ${ }^{25}$

One should note that the effects of finite curvature and cylindrical geometries on the wetting transitions were theoretically addressed by Gelfand et al. ${ }^{26}$ and Bieker et al., ${ }^{27}$ unfortunately however, considering only the opposite curvature at the outer surface. Contrary to the curvature effects studied at the outside walls of cylinders and spheres, in the present case, the interfacial area between the ordered and the disordered phase decreases as the disordered layer becomes thicker. As a consequence, the pore curvature acts to enhance the thickening of the disordered layer. Moreover, wetting of Ising-type systems on spherical or cylindrical surfaces exhibits a different surface phase diagram compared to systems with a flat substrate ${ }^{26}$ Considering the effect of the curvature for spherical and cylindrical geometries on a wetting transition which is first order on a planar substrate, Bieker et al. ${ }^{27}$ demonstrate that at low temperatures, there is no apparent difference between wetting of planar and cylindrical geometries but a dramatic change in wetting scenario occurs near the critical temperature.

\section{CONCLUSION}

In conclusion, we measured the magnetic phase transition of $\mathrm{MnO}$ nanoparticles in confined geometry by means of polarized neutron scattering. We observed the rounding of the magnetic phase transition of $\mathrm{MnO}$ nanoparticles, in contrast to the first-order transition of the bulk MnO. Using the full polarization analysis, we concluded that about $60 \%$ of atoms in the $\mathrm{MnO}$ nanoparticle remain disordered at temperatures as low as $10 \mathrm{~K}$. It supports the suggestion that the surface of $\mathrm{MnO}$ nanoparticles is disordered due to interactions with glass walls. It also can explain why the magnetic correlation length is smaller than the actual size of $\mathrm{MnO}$ nanoparticles, as it was reported by Golosovsky et al. ${ }^{3} \mathrm{We}$ stress that the experimental observation of the lowtemperature magnetic disorder in $\mathrm{MnO}$ nanoparticles was possible only because of the advantage of polarized neutron scattering.

In order to model our experimental data, we applied the theory of surface-induced disorder for semi-infinite systems to describe the wetting of $\mathrm{MnO} / \mathrm{glass}$ interface. Our model is in close agreement with the data and it is based on only one free parameter. While we expect that our model resembles the essential physics for further improvements, the aspect of curvature as well as the Heisenberg spin degrees of freedom 
should also be taken into account. A closer theoretical description is certainly highly desirable since the magnetism on nanoscale is one of the most rapidly developing areas of research.

\section{ACKNOWLEDGMENTS}

We gratefully acknowledge comments from K. Binder and S. Dietrich.
${ }^{1}$ R. H. Kodama, J. Magn. Magn. Mater. 200, 359 (1999).

${ }^{2}$ A.-H. Lu, E. L. Salabas, and F. Schüth, Angew. Chem., Int. Ed. 46, 1222 (2007).

${ }^{3}$ I. V. Golosovsky, I. Mirebeau, G. Andre, D. A. Kurdyukov, Yu. A. Kumzerov, and S. B. Vakhrushev, Phys. Rev. Lett. 86, 5783 (2001).

${ }^{4}$ E. M. Moreno, M. Zayat, M. P. Morales, C. J. Serna, A. Roig, and D. Levy, Langmuir 18, 4972 (2002).

${ }^{5}$ J. F. Löffler, H.-B. Braun, and W. Wagner, Phys. Rev. Lett. 85, 1990 (2000).

${ }^{6}$ J. W. Taylor, J. A. Duffy, C. S. Steer, and C. Shenton-Taylor, J. Magn. Magn. Mater. 310, 2327 (2007).

${ }^{7}$ R. Lipowsky, Phys. Rev. Lett. 49, 1575 (1982).

${ }^{8}$ R. Lipowsky, Z. Phys. B: Condens. Matter 51, 165 (1983).

${ }^{9}$ D. M. Kroll and R. Lipowsky, Phys. Rev. B 28, 6435 (1983).

${ }^{10}$ R. Lipowsky, Ferroelectrics 73, 69 (1987).

${ }^{11}$ W. Schweika, D. P. Landau, and K. Binder, Phys. Rev. B 53, 8937 (1996).

${ }^{12}$ Surface-induced disorder can be interpreted as a critical wetting phenomenon. For critical wetting, temperature $T$ and surface field $h_{s}$ scale in the same way since one can cross the wetting transition line $h_{s}=h_{s, c}(T)$ either by variation in $T$ or in $h_{s}$.

${ }^{13}$ P. Levitz, G. Ehret, and S. K. Sinha, J. Chem. Phys. 95, 6151
(1991).

${ }^{14}$ W. Schweika and P. Böni, Physica B 297, 155 (2001).

${ }^{15}$ V. Privman and M. E. Fisher, J. Appl. Phys. 57, 3327 (1985).

${ }^{16}$ V. Privman and M. E. Fisher, Phys. Rev. B 30, 322 (1984).

${ }^{17}$ J. Hjøllum, Ph.D. thesis, University of Copenhagen, 2008.

${ }^{18}$ J. M. D. Coey, Phys. Rev. Lett. 27, 1140 (1971).

${ }^{19}$ E. Bus and J. A. van Bokhoven, J. Phys. Chem. C 111, 9761 (2007).

${ }^{20}$ J. W. Cahn and J. C. Hilliard, J. Chem. Phys. 28, 258 (1958).

${ }^{21}$ C. G. Shull, W. A. Strauser, and E. O. Wollan, Phys. Rev. 83, 333 (1951)

${ }^{22}$ C. S. Yoo, B. Maddox, J.-H. P. Klepeis, V. Iota, W. Evans, A. McMahan, M. Y. Hu, P. Chow, M. Somayazulu, D. Haüsermann, R. T. Scalettar, and W. E. Pickett, Phys. Rev. Lett. 94, 115502 (2005).

${ }^{23}$ H. Shaked, J. Faber, Jr., and R. L. Hitterman, Phys. Rev. B 38, 11901 (1988).

${ }^{24}$ C. Hock, M. Schmidt, R. Kuhnen, C. Bartels, L. Ma, H. Haberland, and B. v. Issendorff, Phys. Rev. Lett. 103, 073401 (2009).

${ }^{25}$ M. Oguni, S. Maruyama, K. Wakabayashi, and A. Nagoe, Chem. Asian J. 2, 514 (2007).

${ }^{26}$ M. P. Gelfand and R. Lipowsky, Phys. Rev. B 36, 8725 (1987).

${ }^{27}$ T. Bieker and S. Dietrich, Physica A 252, 85 (1998). 